\title{
๙-Fetoprotein-Producing Early Gastric Cancer: Report of Two Cases
}

\author{
KEISHIRO AOYAGI, KIKUO KOUFUJI, SHOJIRO YANO, MOTOSHI MIYAGI, \\ ATSUHIKO KOGA, JINRYO TAKEDA AND KAZUO SHIROUZU
}

Department of Surgery, Kurume University School of Medicine, Kurume 830-0011, Japan

\begin{abstract}
Summary: We have experienced two cases of $\alpha$-fetoprotein (AFP)-producing early gastric cancer. One patient was a 73-year-old man diagnosed as having an early gastric cancer type $0 \mathrm{I}+\mathrm{ll}$ a at $40 \times 40 \mathrm{~mm}$ on the greater curvature of the lower body of the stomach. The histological findings showed that proliferation of a well-differentiated tubular adenocarcinoma with hepatoid pattern was massively invading to the middle layer of the submucosa, with positive lymph vessel, and lymph node metastasis. The other patient was a 76-year-old man diagnosed as having an early gastric cancer type $0 \mathrm{Ila}+\mathrm{llc}$ at $25 \times 25 \mathrm{~mm}$ on the anterior wall on the greater curvature of the antrum. The histological findings showed that proliferation of a small cell carcinoma was massively invading to the deep layer of the submucosa, with positive lymph vessel, and lymph node metastasis. AFP was immunohistochemically found in the tumor cells of these two cases. Both patients died from liver metastasis. AFP-producing early gastric cancer was concluded to be at high risk of liver metastasis.
\end{abstract}

Key words early gastric cancer, AFP-producing gastric cancer, liver metastasis, hepatoid picture, small cell carcinoma

\section{INTRODUCTION}

Early gastric carcinoma is defined as a carcinoma limited to within the mucosa or submucosa of the stomach with or without lymph node metastasis, according to the Guidelines of the Japanese Gastric Cancer Association for Gastric Cancer [1]. The prognosis of early gastric cancer is good. And recurrence or distant metastasis from early gastric cancer is rare. The most frequent site of distant metastasis from early gastric cancer is the liver $[2,3]$.

Recently, $\alpha$-fetoprotein (AFP)-producing gastric cancer is thought to be at high risk of liver metastasis [4-7]. AFP-producing early gastric cancer has been rare. Here, we present two such rare cases of AFPproducing submucosal gastric cancer with fatal metachronous liver metastasis.

\section{CASE REPORT}

Case 1

A 73-year-old man visited Kurume University Hospital complaining of epigastric pain on 22 December 1987. Gastric cancer was diagnosed on Xray examination and upper gastrointestinal endoscopic examination. The patient was admitted for further examination and treatment on 22 January 1988. On admission, the liver, spleen, and tumor were not palpable on physical examination. An X-ray examination and upper gastrointestinal endoscopic examination showed a type $0 \mathrm{IIa}+\mathrm{IIC}$ tumor on the

Received for publication January 4, 2003

Reprint request: Dr. Keishiro Aoyagi, Department of Surgery, Kurume University School of Medicine, 67 Asahi-machi, Kurume 830-0011, Japan. Tel: 81-942-35-3311 Fax: 81-942-34-0709 E-mail: keishiro@med.kurume-u.ac.jp

Abbreviations: AFP, $\alpha$-fetoprotein; CA, carbohydrate antigen; CDDP, cis-diaminodichloroplutinum; CEA, carcinoembryonic antigen; CT, computed tomography. 
greater curvature of the lower body of the stomach. The pathological diagnosis based on a biopsy speci-

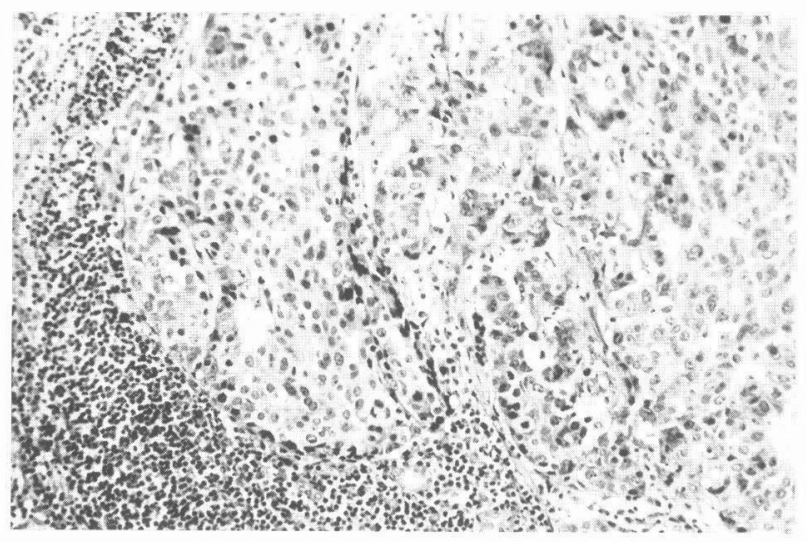

Fig. 1. Solid pattern of tumor cells which had a large nucleus with clear or eosinophilic cytoplasm was recognized in a lymph node $(\mathrm{H} \& \mathrm{E} \times 200)$.

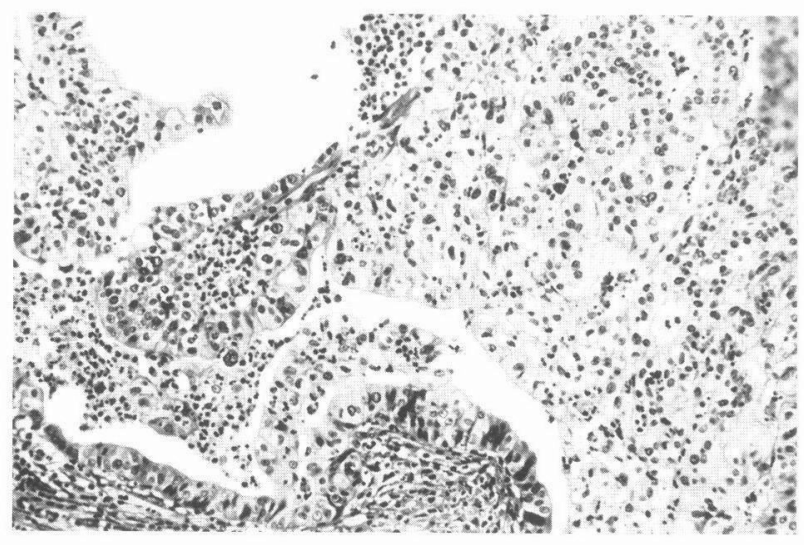

Fig. 2. Trabecular or papillary pattern of tumor cells which had a large nucleus with clear cytoplasm $(\mathrm{H} \& \mathrm{E} \times 200)$.

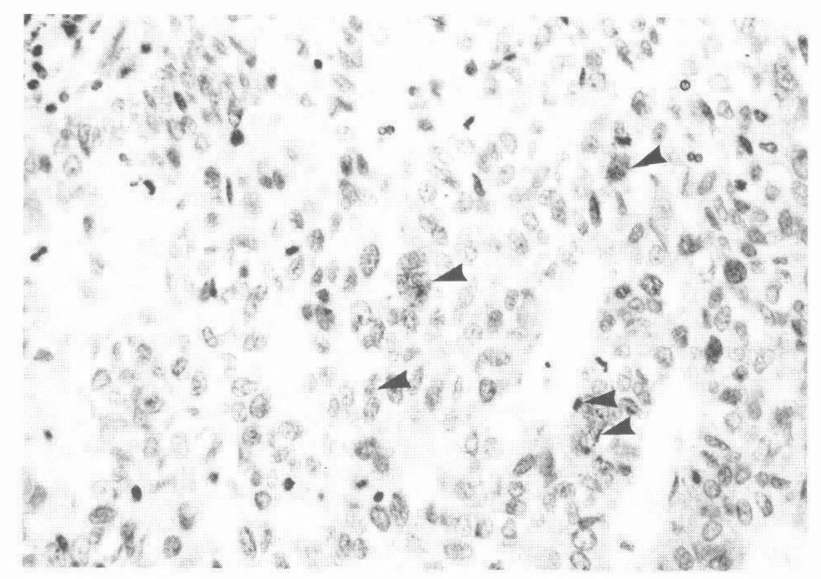

Fig. 3. The tumor showed positive staining with AFP by immunohistochemical staining (arrows) $(\mathrm{AFP} \times 400)$ men from the tumor lesion was a well-differentiated type of tubular adenocarcinoma. The serum levels of carcinoembryonic antigen (CEA) and AFP were normal. No liver metastasis, no lymph node metastasis and no ascites were evident on abdominal ultrasonography. Surgery was performed through a median laparotomy on 9 February. The operative procedure was distal gastrectomy, with D2 lymph node dissection, and Billroth-I reconstruction. The primary tumor was not palpable on the greater curvature of the lower body of the stomach. Macroscopic metastasis was found in the No. 4d lymph node, and not found in the liver and peritoneum. The surgical diagnosis was a gastric cancer type $0 \mathrm{I}$ and $\mathrm{H} 0, \mathrm{P} 0, \mathrm{~N} 1$, T1 Stage IB. The macroscopic findings from the resected specimen were an irregular shaped, protruded and elevated lesion type $0 \mathrm{I}+\mathrm{II} a 40 \times 40 \mathrm{~mm}$ in size on the greater curvature of the lower body of the stomach. The histological findings showed proliferation of a well-differentiated tubular adenocarcinoma massively invading to the middle layer of the submucosa, with positive lymph vessel. Metastases were recognized in five No. $4 \mathrm{~d}$ lymph nodes, histologically (Fig. 1). A hepatoid pattern was recognized in the tumor (Fig. 2). AFP was found in some cancer cells of the hepatoid carcinoma, immunohistochemically (Fig. 3). The patient was discharged from hospital on 1 March. Later, liver and Virchow lymph node metastases were recognized, and he was readmitted on January 1989. At 13 months after surgery, the patient died of liver metastases and Virchow lymph node metastases on 16 March 1989.

Case 2

A 76-year-old man visited a private hospital for a routine screening examination on 27 September 2000. Malignant lymphoma was diagnosed on a biopsy specimen. The patient was referred to Kurume University Hospital for further examination and treatment on 10 October. An X-ray examination and upper gastrointestinal endoscopic examination showed a type 2 tumor on the anterior wall of the greater curvature of the antrum (Fig. 4). The pathological diagnosis based on a biopsy specimen from the tumor lesion was a poorly-differentiated adenocarcinoma. On admission, the liver, spleen and tumor were not palpable on physical examination. The serum levels of carbohydrate antigen (CA 19-9) and CEA were normal. No liver metastasis, no lymph node metastasis and no ascites were evident on computed tomography (CT). Surgery was performed through a median laparotomy on 8 November. The 


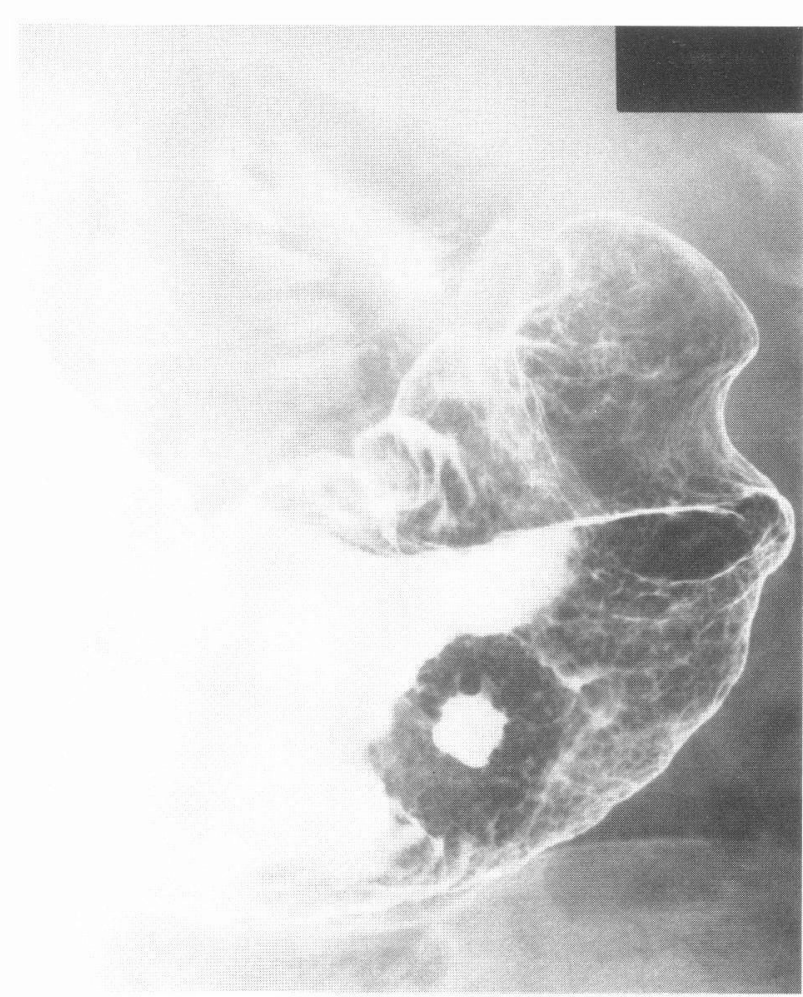

Fig. 4. Double-contrast radiography of the antrum showed an irregular round ulcerative lesion surrounding an irregular protrusion on the anterior wall of the greater curvature.

operative procedure was distal gastrectomy, with D2 lymph node dissection, and Billroth-I reconstruction. The primary tumor was palpable on the anterior wall of the antrum, and no exposed tumor was seen on the serosa. Macroscopic metastasis was found in the No. $4 \mathrm{~d}$ lymph nodes, and not found in the liver and peritoneum. The surgical diagnosis was a gastric cancer type 2 and H0, P0, N1, T2 Stage II. The macroscopic finding from the resected specimen was an ulcerated lesion with sharply demarcated type 0 IIa + IIc $25 \times$ $25 \mathrm{~mm}$ in size on the anterior wall of the greater curvature of the antrum. The histological finding showed proliferation of small cell carcinoma massively invading to the deep layer of the submucosa, with positive lymph vessel (Fig. 5). The tumor showed positive staining with Glimelius stain and with Chromogranin stain. Metastases were recognized in two No. 4d lymph nodes, histologically. AFP was recognized in some cancer cells of the small cell carcinoma, immunohistochemically (Fig. 6). The final diagnosis was a gastric cancer type $0 \mathrm{IIa}+$ IIc and H0, P0, N1, T1 Stage IB. The patient was discharged from hospital on 23 November. Later, liver metastasis was recognized, and he was readmitted in July 2001. Intrahepatic arterial infusion

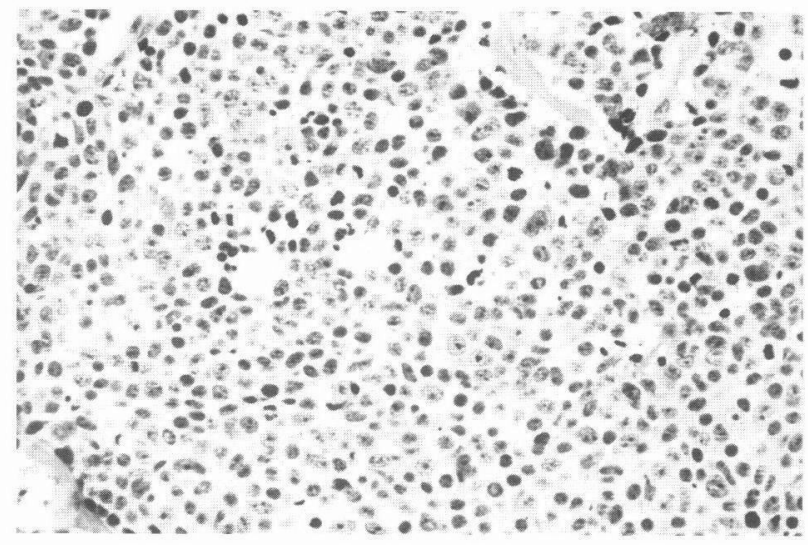

Fig. 5. Medullary and irregular infiltration by tumor cells with little cytoplasm and with round or spindle-shaped chromatin rich nuclei and mitotic figures were seen $(\mathrm{H} \& \mathrm{E} \times 200)$.

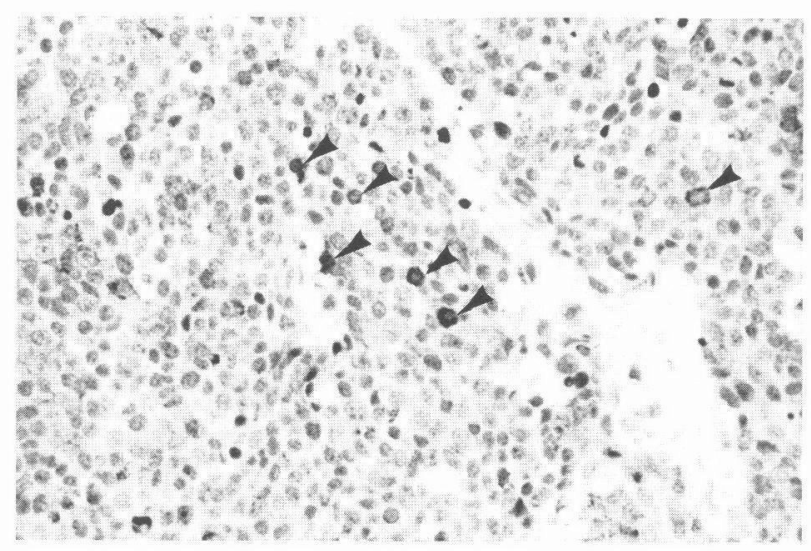

Fig. 6. The tumor showed positive staining with AFP by immunohistochemical staining (arrows) $($ AFP $\times 200)$.

of Cis-Diaminodichloroplutinum (CDDP) was performed, but at 11 months after surgery, the patient eventually died from liver metastases on 12 October 2001 .

\section{DISCUSSION}

Recurrence after curative resection of early gastric cancer is rare, with liver metastasis showing the highest incidence of recurrence [2,3]. Many cases of AFP-producing gastric cancer are advanced cases, and the prognosis is poor [5]. A relationship between liver metastasis and AFP-producing gastric cancer has often been indicated [4-8]; however, the relationship between liver metastasis and AFP-producing early gastric cancer is not clear due to the small number of patients. Ishihara et al. [7] reported that AFP-producing early gastric cancer is characterized 
as submucosal carcinoma, differentiated adenocarcinoma (so-called hepatoid adenocarcinoma) and a high incidence of lymph node involvement. The present two cases were a submucosal carcinoma with lymph node metastasis, and one of them was a welldifferentiated adenocarcinoma with hepatoid pattern, while the other case was a small cell carcinoma. The histological feature of a hepatoid adenocarcinoma is a solid or trabecular pattern of tumor cells which have a large nucleus with clear or eosinophilic cytoplasm, similar to the pattern of hepato cellular carcinoma [9]. Liver metastasis from a hepatoid adenocarcinoma in the stomach is frequently recognized, and many cases of hepatoid adenocarcinomas are AFPproducing tumors [9]. The histological feature of a small cell carcinoma is a cluster of atypical cells with large pleomorphic-shaped nuclei which are arranged homogenously in sheets, and mitotic figures of tumor cells are frequent [10]. Tumor cells of small cell carcinoma have shown positive staining with Glimelius stain, and neurosecretary granules have been recognized in the cytoplasm of tumor cells of small cell carcinoma on electron microscopy $[11,12]$. The prognosis of small cell carcinoma is very poor [13]. Ishihara et al. [7] reported that the relationship between the liver metastasis and AFP-producing tumor in early gastric cancer patients with liver metastasis appears meaningful. Chang et al. [5] reported that AFP-producing early gastric cancer has the same tendency for liver metastasis as AFP-producing advanced gastric cancer. It is important to detect patients with early gastric cancer who will develop liver metastasis. To prevent recurrence, early diagnosis by immunohistochemical staining of AFP for specimens with a hepatoid picture or small cell carcinoma, and aggresive chemotherapy are mandatory.

\section{REFERENCES}

1. Japanese Gastric Cancer Association. The General Rules for Gastric Cancer Study, 13th edn, Kanehara, Tokyo, p5-6, 1999. (in Japanese)

2. Nishi $M$, Ishihara $S$, and Nakajima T. Chronological changes of characteristics of early gastric cancer and therapy: experience in the Hospital of Tokyo Cancer Institute 1950-1994. J Cancer Res Clin Oncol 1995; 121:535-541.

3. Aoyagi K, Koufuji K, Yano S, Murakami N, Hori H et al. A study on recurrent cases of submucosal gastric cancer. Jpn J Clin Exp Med (Rinsho to Kenkyu) 1999; 76:1783-1785. (in Japanese)

4. Takahashi Y, Mai M, Akimoto R, Ogino T, Ueda $\mathrm{H}$ et al. Clinicopathological study on high risk cases of liver metastasis from gastric cancer-with reference to AFPproducing tumor of the stomach Jpn J Gastroenterol Surg (Nihon Shoukaki Geka Gakkai Zasshi) 1984; 17:17321736. (in Japanese)

5. Chang YC, Nagasue N, Abe S, Kohno H, Yamanoi A et al. The characters of AFP-producing early gastric cancer. J Jpn Surg Soc (Nihon Geka Gakkai Zasshi) 1990; 91:1574-1580. (in Japanese)

6. Tsurumachi $\mathrm{T}$, Yamamoto $\mathrm{H}$, Watanabe $\mathrm{K}$, Honda I, Watanabe $\mathrm{S}$ et al. Resection of liver metastasis from alpha-fetoprotein-producing early gastric cancer: report of a case. Surg Today 1997; 27:563-566.

7. Ishihara $\mathrm{S}$, Yanagisawa $\mathrm{A}$, and Takahashi $\mathrm{T}$. Clinicopathologic and immunohistochemical study on $\alpha$ fetoprotein-producing early gastric cancer with liver metastasis. Jpn J Gastroenterol Surg (Nihon Shoukaki Geka Gakkai Zasshi) 1999; 32:2314-2319. (in Japanese)

8. Kimura O, Yurugi E, Okamoto $T$, Nishidoi $H$, Takebayashi $\mathrm{M}$ et al. A histopathological study of gastric cancer with liver metastasis, with special reference to poorly differentiated adenocarcinoma of medullary type. Jpn J Cancer Clin (Gann no Rinsho) 1984; 30:131-137. (in Japanese)

9. Ishikura $\mathrm{H}$, Kirimoto $\mathrm{K}$, Shamoto $\mathrm{U}$, Miyamoto $\mathrm{Y}$, Yamagiwa $\mathrm{H}$ et al. Hepatoid adenocarcinomas of the stomach-an analysis of seven cases. Cancer 1986; 58:119-126.

10. Matsusaka T, Watanabe H, and Enjoji M. Oat-cell carcinoma of the stomach. Fukuoka Acta Med 1976; 67:6573.

11. Kaketani K, Mitarai Y, Zeze K, Kuwahara A, Saito T et al. Two cases of oat-cell carcinoma of the stomach. J Jpn Surg Assoc (Nihon Rinsho Geka Gakkai Zasshi) 1987; 48:1687-1692. (in Japanese)

12. Iwabuchi $\mathbf{M}$, Ishihara $\mathbf{N}$, and Watanabe $\mathrm{H}$. Histogenesis of endocrine cell carcinoma of the stomach. Jpn J Cancer Clin (Gann no Rinsho) 1984; 30:435-437. (in Japanese)

13. Aoyagi K, Kohfuji K, Yano S, Murakami N, Hori H et al. Primary small cell carcinoma of the stomach: report of a case. Stomach and Intestine (I to Cho) 2000; 35:246250. (in Japanese) 